\section{RCA: A program for regression component analysis}

\section{ROLF LANGEHEINE and HOLGER SÖNNICHSEN \\ Institute for Science Education, University of Kiel D-2300 Kiel 1, West Germany}

Factor analysis has been a popular data reduction method for some time, especially but not exclusively in the social sciences, notwithstanding the fact that the underlying conventional factor model (CFM) is beset with several conceptual problems that have been known, at least in principle, since its inception (e.g., Wilson, 1928). Foremost among these is the peculiar fact that the CFM

$$
\begin{aligned}
& Y=X A^{\prime}+Z U, X^{\prime} X /(N-1)=\Psi, \\
& \left.Z^{\prime} Z / N-1\right)=I_{p}, X^{\prime} Z /(N-1)=\phi
\end{aligned}
$$

(where $Y, N x p$ contains the observed deviation scores for $\mathrm{N}$ subjects on $\mathrm{p}$ tests and $\mathrm{X}, \mathrm{Nxm}$ and $\mathrm{Z}, \mathrm{Nxp}$, the common and unique factor scores, and $A, U$ contain regression weights) does not permit the construction of the factor score matrices $X$ and $Z$ as linear combinations of the observed scores in $Y$ (see, e.g., Schönemann \& Steiger, 1976). To construct $X$ and $Z$, it is necessary, instead, to invoke an essentially arbitrary matrix of random numbers. As a consequence, $X$ and $Z$ are indeterminate within a wide margin. This so-called "factor indeterminacy problem" has a number of conceptual ramifications that go beyond the immediate difficulty of selecting one of many possible pairs of such matrices $X$ and $Z$. They becloud the interpretation and logical status of the results obtained with this model quite generally.

Many users, however, are unconcerned, if not unaware of these difficulties. The reason is that their main interest is not so much in the CFM per se but in the purely pragmatic objective of reducing a given number of $p$ variables (in $Y$ ) to a more manageable, smaller number of new variables. The latter hopefully lend themselves to more transparent psychological interpretation. To this end, users often compute, at the conclusion of a factor analysis, "factor score estimates," which are then used in further research. These "estimates" are, of course, computed as linear combinations of the observed variables in $Y$, and thus they are better called "components," to distinguish them from factors. Moreover, they are in most cases virtually indistinguishable from scores obtained with principal component analysis (PCA; Hotelling, 1933) after they have been standardized and rotated to simple structure.

The present program combines a variety of computational options for obtaining such components within the somewhat more general framework of regression component analysis (RCA; Schönemann \& Steiger, 1976), which has been formulated in an attempt to emulate the typical factor-analytic procedures without incurring the conceptual problems of the underlying model. Although not restricted to PCA, RCA contains it as a special case.

The defining equations of RCA are:

$$
Y=X A^{\prime}+E, X=Y B, A=\operatorname{cov}(Y, X) \operatorname{var}^{-1}(X) \text {. }
$$

Thus, the observed deviation score matrix $Y$ is decomposed, as it is in factor analysis, into a sum of two parts, $\hat{\mathrm{Y}}=\mathrm{XA}^{\prime}$ and $\mathrm{E}$, which, as a consequence of Equation 2, are pairwise uncorrelated, as they are in factor analysis. The matrix $\mathbf{A}$, which is used to approximate the observed scores $Y$, is again, as in factor analysis, a matrix of regression weights. The main and decisive difference, however, is the definition of $X$ : These scores are explicitly defined as linear combinations of those in $\mathrm{Y}$ and, thus, completely determinate by definition. Matrix B is therefore called the "matrix of defining weights," and the Matrix A is called the "regression pattern." The defining relations (Equation 2) imply a 1:1 relationship between $A$ and $B$ :

$$
A=C B\left(B^{\prime} C B\right)^{-1}, B=C^{-1} A\left(A^{\prime} C^{-1} A\right)^{-1} .
$$

This means that an RCA can be performed either on the basis of a regression pattern $A$ or, alternatively, on the basis of a matrix of defining weights $B$. For example, the defining weights might be obtained through a canonical correlation analysis or a linear discriminant analysis, or the regression pattern might be obtained, for purposes of comparison, through conventional factor analysis. More useful and typical, though, might be a regression pattern obtained from PCA, especially after the retained components have been standardized to preserve their stochastic independence under all orthogonal rotations. If the eigen decomposition of the observed covariance (or correlation) matrix is

$$
C=Y^{\prime} Y /(N-1)=L_{1} D_{1}^{2} L_{1}^{\prime}+L_{2} D_{2}^{2} L_{2}^{\prime}
$$

(where $D_{1}^{2}$ contains the $m$ largest latent roots and $L_{1}$ contains the associated eigen vectors), then the regression pattern (prior to rotation) is simply

$$
A=L_{1} D_{1},
$$

and the defining weights are

$$
\mathrm{B}=\mathrm{L}_{1} \mathrm{D}_{1}^{-1} \text {. }
$$

This type of data reduction via PCA is well-known 
and has been widely used, although it sometimes has been confused with factor analysis proper, especially since the numerical outcome of both types of data analysis tend to be quite similar (e.g., Kallina \& Hartmann, 1976; Velicer, 1972). The theoretical difference, though, is considerable: In factor analysis we deal, strictly speaking, with a falsifiable model, whereas such a component analysis is simply a tautological data reduction. On the other hand, the variables of the factor model are indeterminate, whereas such components, by definition, are well defined linear functions of the observed variables in Y. For purposes of data reduction, it would seem that such a component analysis is the preferred choice because it steers clear of the indeterminacy problems of the CFM.

The present program performs such an RCA. It has several input options and also provides a number of popular options for further processing and redefining the regression pattern, for example, through orthogonal or oblique rotation to simple structure or orthogonal or oblique target rotation ("procrustes" fit). The program further computes the full set of component scores $\mathrm{X}=\mathrm{YB}$ for the matrix of defining weights that correspond to the regression pattern that has been decoded. For flexibility, the program permits the user to commence the computational sequence with a matrix of defining weights $B$ rather than $A$. So the user can then compute the associate regression pattern $A$, for example, for purposes of comparison with other analogous data analysis procedures, such as factor analysis proper.

Limitations. The present version of RCA accepts data for up to 35 variables with no restriction in number of cases. Redimensioning is easy due to dynamic subroutine dimensioning. This version requires a core storage of roughly $42 \mathrm{~K}$ for loading and roughly $35 \mathrm{~K}$ for running.

Programming Language. FORTRAN IV is the language used. The program has been written and checked on a DECsystem 10.

Availability. The program is available from Rolf Langeheine, IPN at the University of Kiel, Olshausenstrasse 40-60, D-2300 Kiel 1, Federal Republic of Germany. Detailed documentation is given in the comments of the program. Copies may be loaded on your magnetic tape or DECtape free of charge.

\section{REFERENCES}

Hotelling, H. Analysis of a complex of statistical variables into principal components. Journal of Experimental Psychology, $1933,24,417-442 ; 498-520$.

Kallina, H., \& Hartmann, A. Ein Verlgeich von Hauptkomponentenanalyse und klassischer Faktorenanalyse. Psychologische Beiträge, 1976, 18, 84-98.

Schönemann, P. H., \& Steiger, J. H. Regression component analysis. British Journal of Mathematical and Statistical Psychology, 1976, 29, 175-189.

VELICER, W. An empirical comparison of factor analysis, image analysis, and principal component analysis. Unpublished doctoral dissertation, Purdue University, 1972.

WiLson, E. B. Review of "The abilities of man, their nature and measurement" by C. Spearman. Science, 1928, 67, 244-248.

(Accepted for publication September 6, 1981.) 